\title{
THE FUNDAMENTAL GROUP OF THE PRINCIPAL COMPONENT OF A COMMUTATIVE BANACH ALGEBRA ${ }^{1}$
}

\author{
EDWARD K. BLUM
}

We consider an arbitrary commutative Banach algebra over the complex numbers. Let $B$ denote the algebra, $\{a, b, c, x, y, z, \cdots\}$ its elements, and $\{\lambda, \mu, \nu, \cdots\}$ complex numbers. We assume that $B$ contains a unit element, $e$, with $\|e\|=1$.

If $a^{-1}$ exists ( $a a^{-1}=a^{-1} a=e$ ), the element $a$ is called "regular." The set of regular elements will be denoted by $G$. It is well known that (1) $G$ is a topological group relative to multiplication and (2) $G$ is an open subset of $B$. Since $G$ is open, it is a union of maximal open connected sets, its components. We call the component $G_{1}$ containing the unit $e$ the "principal component" [1]. It is easy to see that $G_{1}$ is a subgroup of $G$.

The function $\exp (x) \equiv e+\sum_{1}^{\infty} x^{n} / n$ ! is defined for all $x$ in $B$ and has the usual properties of the classical exponential function. If we let $\pi_{1}\left(G_{1}\right)$ denote the fundamental group of $G_{1}$, we may state our main result as follows:

Theorem 1. Let $P=\{x \mid \exp (x)=e\}$. $P$ is an additive group which is isomorphic to $\pi_{1}\left(G_{1}\right)$.

We shall give a complete proof based on Schreier's theory of the universal covering group and then we shall outline a second proof which depends only on results from the theory of Banach algebras. ${ }^{2}$ The result of Schreier which we shall use may be stated as follows[2]:

THEOREM. Let $B$ be a simply-connected, locally-connected and locally simply-connected topological group. If $P$ is a discrete normal subgroup of $B$, then the fundamental group of the topological space $B / P$ is isomorphic to the group $P$.

The algebra $B$, regarded as an additive group with the metric topology of the norm, clearly satisfies the hypotheses of Schreier's

Presented to the Society, February 28, 1953; received by the editors September 10, 1952.

1 This result is contained in the author's doctoral dissertation, The theory of analytic functions in Banach algebras, completed in June 1952 under E. R. Lorch at Columbia University.

2 The second proof is the one used in the author's dissertation. It was later pointed out by S. Eilenberg that a different proof is possible if one brings the Schreier theory to bear. 
theorem. The set $P=\{x \mid \exp (x)=e\}$ is obviously a normal subgroup of $B$ since $\exp (x-y)=\exp (x) \exp (-y)=\exp (x)[\exp (y)]^{-1}$. Further, $\exp (x)$ maps $B$ onto the principal component, $G_{1}$ [3]. It is clearly a continuous map. It is also an open mapping since its inverse, $\log y$, is continuous. Thus, $\exp (x)$ is an open homomorphism of the additive topological group $B$ onto the multiplicative topological group $G_{1}$. The kernel of this homomorphism is $P$, so that $B / P$ is isomorphic to $G_{1}$ as topological groups. Hence $\pi_{1}(B / P)$ is isomorphic to $\pi_{1}\left(G_{1}\right)$. If it can be shown that $P$ is discrete, the Schreier theorem is applicable and Theorem 1 follows immediately.

To prove $P$ discrete it suffices to show that 0 is an isolated element of $P$. Suppose there is a sequence of elements $\left\{z_{n}\right\}$ such that $z_{n} \in P, z_{n}$ $\neq 0$ and $\lim z_{n}=0$. By a theorem of Lorch [1], this implies that $z_{n}=2 \pi i \sum_{j=1}^{k} n_{j} e_{j}$, where the $e_{j}$ are idempotent elements $\left(e_{j}^{2}=e_{j}\right)$ and the $n_{j}$ are rational integers. Furthermore, the spectrum of $z_{n}$ consists of the points $2 \pi i n_{1}, \cdots, 2 \pi i n_{k}$. However, $\lim \left\|z_{n}\right\|=0$ and, as is well known, the spectrum of $z_{n}$ contains no points exterior to the circle of radius $\left\|z_{n}\right\|_{1}$, center at the origin. For sufficiently large $n$, this means that $n_{j}=0$ for $j=1, \cdots, k$; i.e. $z_{n}=0$. This contradiction completes the proof.

Now we shall indicate a more elementary and constructive proof in which no recourse is had to the Schreier theory.

First we establish a lemma concerning the function $\exp (x)$.

LEMMA: Let $w_{0}=\exp \left(x_{0}\right)$ and $0<\epsilon<1 /\left\|w_{0}^{-1}\right\|$. Let

$$
\delta=\sum_{1}^{\infty} \frac{\left(\left\|w_{0}^{-1}\right\| \epsilon\right)^{n}}{n} .
$$

If $E$ is the set $\left\{w \mid\left\|w-w_{0}\right\|<\epsilon\right\}$, then for every $w$ in $E$ there is an $x$ such that $\left\|x-x_{0}\right\|<\delta$ and $\exp (x)=w$.

Proof. Choosing $w=w_{0}+b$ where $\|b\|<\epsilon$, we have $\left\|w_{0}^{-1} w-e\right\|$ $=\left\|w_{0}^{-1} b\right\| \leqq\left\|w_{0}^{-1}\right\|\|b\|<1$. Hence $w_{0}^{-1} w$ is in $G_{1}$ and there is an element $c$ in $B$ such that $\exp (c)=w_{0}^{-1} w$. In fact, we may take

$$
c=\sum_{1}^{\infty}-(1 / n)\left(e-w_{0}^{-1} w\right)^{n}
$$

so that $\|c\|<\sum_{1}^{\infty}(1 / n)\left(\left\|w_{0}^{-1}\right\| \epsilon\right)^{n}=\delta$. The element $x=x_{0}+c$ satisfies the conclusion of the lemma. It is important to note that $\delta$ approaches 0 as $\epsilon$ approaches 0 .

Using this lemma, we are able to prove

THEOREM 2. Let $K:\{f(s), 0 \leqq s \leqq 1\}$ be a curve in $G_{1}$ joining $e=f(0)$ 
to $w=f(1)$. There exists an element $z$ in $B$ such that $\exp (z)=w$ and the curve $K(z):\{\exp (t z), 0 \leqq t \leqq 1\}$ is homotopic to $K$ in $G_{1}$.

Proof. If $z_{s}$ is such that $\exp \left(z_{s}\right)=f(s)$, let $K\left(z_{s}\right)$ denote the curve $\left\{\exp \left(t z_{s}\right), 0 \leqq t \leqq 1\right\} . K\left(z_{s}\right)$ is a compact set in $G_{1}$. Hence, there is a number $\rho>0$ such that every sphere with center on $K\left(z_{s}\right)$ and radius $\rho$ is contained in $G_{1}$. Choose $\epsilon$ such that $0<\epsilon<\min \{\rho, 1\}$. There is a number $\gamma(\epsilon)>0$ such that $\|f(r)-f(s)\|<\epsilon$ whenever $|r-s|<\gamma(\epsilon)$. By the lemma, there is an element $z_{r}$ such that $\left\|z_{r}-z_{s}\right\|<\delta$ and $\exp \left(z_{r}\right)$ $=f(r)$, where $\delta=\sum_{1}^{\infty}(1 / n)\left(\left\|[f(s)]^{-1}\right\| \epsilon\right)^{n}$, that is, $z_{r}=z_{s}+b$ where $\|b\|<\delta$.

For all $t, 0 \leqq t \leqq 1,\left\|\exp \left(t z_{r}\right)-\exp \left(t z_{s}\right)\right\| \leqq\left\|\exp \left(t z_{s}\right)\right\| \cdot\|\exp (t b)-e\|$ $\leqq \sum_{0}^{\infty}(1 / n !)\left\|z_{s}\right\|^{n} \cdot \sum_{1}^{\infty}(1 / n !)\|b\|^{n} \leqq \exp \left\|z_{s}\right\| \sum_{1}^{\infty} \delta^{n} / n !$. By choosing $\epsilon$ sufficiently small, thereby making $\delta$ small, we have $\| \exp \left(t z_{r}\right)$ $-\exp \left(t z_{s}\right) \|<\rho$. It follows that $K\left(z_{s}\right)$ is homotopic in $G_{1}$ to the curve $K\left(z_{r}\right) \cup K_{r}^{s}$ consisting of $K\left(z_{r}\right)$ followed by the arc $K_{r}^{s}:\{f(u), r \leqq u \leqq s\}$.

In particular, since exp $(0)=f(0)=e$, there is an $r>0$ and an element $z_{r}$ such that $K\left(z_{r}\right)$ is homotopic to the arc $K_{0}^{r}:\{f(s) \mid 0 \leqq s \leqq r\}$. The set of all real numbers $r$ for which this holds has a least upper bound, $\mu \leqq 1$. Suppose $\mu<1$. We obtain a contradiction.

Let $\exp \left(z_{\mu}^{\prime}\right)=f(\mu)$. There is an element $z_{s}^{\prime}$ such that $K\left(z_{\mu}^{\prime}\right)$ is homotopic to $K\left(z_{s}^{\prime}\right) \cup K_{s}^{\mu}, s<\mu$. But there is a $z_{s}$ such that $K\left(z_{s}\right)$ is homotopic to $K_{0}^{s}$. Since $\exp \left(z_{s}^{\prime}\right)=\exp \left(z_{s}\right)=f(s)$, we have $z_{s}=z_{s}^{\prime}+c$ where $\exp (c)=e$. Let $z_{\mu}=z_{\mu}^{\prime}+c$. It is simple to show that $K\left(z_{\mu}\right)$ is homotopic to $K\left(z_{s}\right) \cup K_{s}$ which is, in turn, homotopic to $K_{0}^{s} \cup K_{s}^{\mu}=K_{0}^{\mu}$. Hence $K\left(z_{\mu}\right)$ is homotopic to $K_{0}^{\mu}$. By the same reasoning, there is a number $s_{1}>\mu$ such that $K_{0}^{s_{1}}$ is homotopic to $K\left(z_{s_{1}}\right)$, contradicting the assumption on $\mu$. Therefore, $\mu=1$.

The next theorem then follows easily.

TheOREM 3. Every closed curve in $G_{1}$ with e as initial and end point is homotopic in $G_{1}$ to a curve $K(b)$ of the form $\{\exp (t b) \mid 0 \leqq t \leqq 1, \exp (b)$ $=e\}$. If $\exp \left(b^{\prime}\right)=e$ and $b \neq b^{\prime}$, then $K\left(b^{\prime}\right)$ is not homotopic to $K(b)$. Thus each homotopy class contains precisely one curve of the form $\{\exp (t b), 0 \leqq t \leqq 1\}$ where $\exp (b)=e$.

Proof. The first part of the theorem is obtained by choosing any point $w=f(s)$ on $K$. By Theorem 2, there is a $z$ such that $K(z)$ is homotopic to $K_{0}^{s}$ and a $z^{\prime}$ such that $K\left(z^{\prime}\right)$ is homotopic to $K_{8}^{1}$. The element $b=z^{\prime}-z$ gives the desired result.

Noting that $\int_{K(b)} x^{-1} d x=b$ and $\int_{K\left(b^{\prime}\right)} x^{-1} d x=b^{\prime}$, we see that $K(b)$ cannot be homotopic to $K\left(b^{\prime}\right)$, for in that event, the integrals would 
be equal by the Cauchy integral theorem (by [1] and a result in the author's dissertation not yet published).

Theorem 1 follows directly from Theorem 3.

\section{BIBLIOGRAPHY}

1. E. R. Lorch, The theory of analytic functions in normed abelian vector rings, Trans. Amer. Math. Soc. vol. 54 (1943) pp. 414-425.

2. L. Pontrjagin, Topological groups, Princeton University Press, 1946, p. 231.

3. M. Nagumo, Einige analytische Untersuchungen in linearen metrischen Ringen, Jap J. Math. (1936).

Columbia University and

UNIVERSITY OF MARYLAND

\section{REMARK ON A FORMULA FOR THE BERNOULLI NUMBERS}

\section{CARLITZ}

Some years ago Garabedian [1] proved the following formula:

$$
B_{k+1}=\frac{(-1)^{k+1}(k+1)}{2^{k+1}-1} \sum_{r=0}^{k}(-1)^{r} \frac{\Delta^{r} 1^{k}}{2^{r+1}} \quad(k \geqq 0),
$$

where the even suffix notation is employed for the Bernoulli numbers. The proof of (1) made use of the sum of a certain divergent series.

We wish to point out that (1) is not new. It can be found (in somewhat different notation) in $[3$, p. 224 , formula (68)].

It may be of interest to give a short proof of (1). We use the formula $[2$, p. 28]

$$
C_{k}=2^{k+1}\left(1-2^{k+1}\right) \frac{B_{k+1}}{k+1},
$$

where the $C_{k}$ are the coefficients in the Euler polynomial:

$$
E_{k}(x)=\left(x+\frac{C}{2}\right)^{k}=\sum_{s=0}^{k}\left(\begin{array}{l}
k \\
s
\end{array}\right) 2^{-s} C_{s} x^{k-s} .
$$

Then in view of

$$
E_{k}(x+1)+E_{k}(x)=2 x^{k},
$$

we have

$$
E_{k}(x)=\left(1+\frac{1}{2} \Delta\right)^{-1} x^{k}=\sum_{s=0}^{k}(-1)^{\cdot} 2^{-s} \Delta^{s} x^{k}
$$

Received by the editors September 5, 1952. 